Scientific Paper

\title{
Nanoscale dosimetric consequences around bismuth, gold, gadolinium, hafnium, and iridium nanoparticles irradiated by low energy photons
}

\author{
Asghar MESBAHI ${ }^{\mathrm{a}, \mathrm{c}}$, Elham MANSOURI ${ }^{\mathrm{b}}$, Mohammad MOHAMMADZADEH ${ }^{\mathrm{c},{ }^{*}}$ \\ ${ }^{a}$ Molecular Medicine Research Center, Biomedicine Institute, Tabriz University of Medical Sciences, Iran \\ ${ }^{b}$ Drug Applied Research Center, Tabriz University of Medical Sciences, Tabriz, Iran \\ ${ }^{c}$ Medical Radiation Sciences Research Team, Tabriz University of Medical Sciences, Iran \\ *E-mail address: radiationoncology2010@gmail.com,mohammadzadehmohammad@yahoo.com
}

\begin{abstract}
In the current study, nanoscale physical dose distributions around five potential nanoparticles were compared. Five potential nanoparticles including bismuth, gold, gadolinium, hafnium, and iridium nanoparticles in the form of a sphere with a diameter of $50 \mathrm{~nm}$ were simulated in a water medium. The MCNPX (2.7.0) Monte Carlo code with updated libraries was used for calculations of electron dose deposition and electron flux in water from $25 \mathrm{~nm}$ up to $4000 \mathrm{~nm}$ with a step of $25 \mathrm{~nm}$. Also, secondary electron spectra after irradiation of nanoparticles with mono-energetic photons with energies of 30,60, $100 \mathrm{keV}$ were derived. The nano-scale distance-dose curves showed a very steep gradient with distance from nanoparticle surface up to $60 \mathrm{~nm}$ and after this point, a gradual decrease was seen. The dose deposition characteristics in the nano-scale were dependent on the type of nanoparticle as well as photon energy. Our results concluded that for each photon energy in the energy range of 30-100 keV, a suitable nanoparticle can be selected to boost the effect of energy deposition by low energy photon beams used in brachytherapy.
\end{abstract}

Key words: nanoparticle; radiation therapy; electron spectra; heavy atoms.

\section{Introduction}

Application of nanoparticles in medical radiation physics, medical imaging, and radiation therapy of cancer has been reported by enormous studies. ${ }^{1-10}$ Several nanoparticles have been used and studied for application in nanoparticle-aided radiation therapy (NART) with the photons in the energy range of tens to hundreds of $\mathrm{keV}^{2,5,11-17}$ The studies showed that photoelectrons produced through photoelectric interaction of photons with high $\mathrm{Z}$ atoms of nanoparticles are the major players in the extra dose delivered around the nanoparticles. ${ }^{15,18}$ However, after the photoelectric phenomenon, in addition to photoelectrons, the auger electrons, characteristics x-rays, other secondary electrons are released from nanoparticles and deliver a high dose near the surface of nanoparticles. ${ }^{18,19}$ The contribution of photoelectrons and auger and other secondary electrons in absorbed dose around a nanoparticle depends strongly on the nanoparticle size, photon energy, and atomic composition of the nanoparticle. Investigations reported that for nanoparticles with a diameter less than $10 \mathrm{~nm}$ the auger electron contribution in absorbed dose can be very significant but for larger nanoparticles, the auger electrons can be highly absorbed inside of nanoparticle and only high energy photoelectrons will be able to deposit their energy at several tens of nanometer from particle surface and are responsible for observed dose enhancement effect. ${ }^{6,20-25}$
As it is evident the secondary electrons produced by photon beams after hitting the nanoparticle are responsible for the dose enhancement effect of NART, thus knowing their dosimetric features in the nano-scale including secondary electron energy spectra, distance-dose relationship, and secondary electrons flux variations with distance from nanoparticle can provide useful information for more optimized NART of cancers. However, it should be noticed here that the final clinical consequence of NART will be dependent on several biochemical and biological phenomena that are beyond the scope of the current paper. Only the radiation physics aspect of the dose deposition effect of some nanoparticles will be studied here.

Numerical studies including analytical and MC studies have shown the importance of photon energy and the atomic number of nanoparticles in dose enhancement factor (DEF). There are many papers on gold and bismuth dose enhancement effect. Also, gadolinium which is used extensively as an MRI contrast agent has been investigated as a radiosensitizer in radiation therapy. ${ }^{11,26,27}$ Moreover, hafnium oxide was used as a radiosensitizer in radiation therapy by several investigations. ${ }^{14,28}$ Several reliable publications state that the total dose enhancement effect of nanoparticles, in reality, is composed of three components of physical, chemical, and biological enhancement. ${ }^{19,22,29-32}$ It can help us to interpret the 
considerable discrepancy observed between calculated DEF and observed experimental DEF for high energy photons in the $\mathrm{MeV}$ range where the photoelectric phenomenon reduces significantly and the Compton effect dominates. On the other hand, if only physical DEF is considered, there are many investigations with a widespread range of nanoparticle size, type, concentration, and photon energy which make the estimation of physical DEF very difficult.

Experimental electron spectroscopy for nanoparticles is a very cumbersome and sophisticated procedure. Thus, Monte Carlo (MC) modeling of single nanoparticle from the different types and for applied photon energy in brachytherapy can give more information on the nano-scale dose estimations.

To the best of our knowledge, there is no comparative study on the dosimetric properties of these nanoparticles in the literature. In the current study, five potential nanoparticles were selected based on the literature and their physical dose deposition was calculated in nano-scale for comparison purposes. We think that the results can provide new information in the selection and the design of alternative nanoparticles for radiation therapy applications. On the other hand, to provide more detailed physical information concerning the potential nanoparticles, optimize DEF, and consequently cell toxicity in conjunction with X-rays, the current study was conducted. In the current study, using MCNPX MC code a single nanoparticle made of bismuth, Gold, Gadolinium, Hafnium, and iridium was simulated and the secondary electron dose, flux, and spectra were calculated for three photon energies of $30,60,100 \mathrm{keV}$. Based on the provided data, the nano-scaled dose deposition of the studied nanoparticles was compared and analyzed.

\section{Materials and Methods}

In the current study, we used MCNPX (2.7.0) Monte Carlo code for scoring electron fluence, electron energy spectra, and dose deposition around a single spherical nanoparticle made of different elements including bismuth, gold, gadolinium, Hafnium, Iridium. The MCNPX code can simulate the transportation and tracking of secondary electrons down to 1 $\mathrm{keV}$ energy. The range of $1 \mathrm{keV}$ electron could be around 10 $\mathrm{nm}$ in water. The simulation geometry was based on the study of Leung et al. ${ }^{24}$ depicted in Figure 1. To ensure the geometry accuracy and monitoring the electron and photon tracks the visual editor of MCNPX code was used. A spherical nanoparticle with a radius of $25 \mathrm{~nm}$ was located at the center of the simulation space. Then, multiple concentric spherical surfaces with $25 \mathrm{~nm}$ increments were simulated around nanoparticle to score electron fluence and provide shell-like cells for dose scoring around it. A circular-planar photon source with a radius of $25 \mathrm{~nm}$ to irradiate the nanoparticle uniformly was defined at the distance of $5 \mathrm{~nm}$ from the nanoparticle. The photons trajectories were set to be parallel and impinging on nanoparticles homogeneously.
Three quantities were tallied for each nanoparticle type and three energies of mono-energetic beams of 30,60, $100 \mathrm{keV}$. The selection of the nanoparticle types was based on the previous studies, which reported potentials and advantages for these nanoparticles. However, iridium with the highest density was selected by this research for the first time due to its atomic number and density. Studied nanoparticles comprised of Bismuth $\left(Z=83, \quad\right.$ density $\left.=9.78 \mathrm{~g} / \mathrm{cm}^{3}\right), \quad$ Gold $\quad(Z=79$, density $\left.=19.32 \mathrm{~g} / \mathrm{cm}^{3}\right)$, Gadolinium $(Z=64$, density $=7.89$ $\left.\mathrm{g} / \mathrm{cm}^{3}\right)$, Hafnium $\left(Z=72\right.$, density $\left.=13.31 \mathrm{~g} / \mathrm{cm}^{3}\right)$, Iridium $\left(Z=77\right.$, density $\left.=22.56 \mathrm{~g} / \mathrm{cm}^{3}\right)$.

For photoelectrons and auger electrons coming out from the nanoparticle, fluence and energy spectra scoring were performed using F2 tally which calculates electron crossing a surface in terms of number per $\mathrm{cm} 2$ for the surface of the nanoparticle as well as other concentric spherical surfaces with radii starting from $50 \mathrm{~nm}$ with an increment of $25 \mathrm{~nm}$ up to $4000 \mathrm{~nm}$. Also, the dose delivered to the peripheral cells around the nanoparticles was calculated using *F8:e tally. for excluding the dose deposition from primary and scattered photons, the importance of photons was set to be zero for all scoring cells except the first cell where the photon source was located. The concentric cells were filled with water with a density of $1 \mathrm{~g} / \mathrm{cm}^{3}$ in dose deposition calculations.

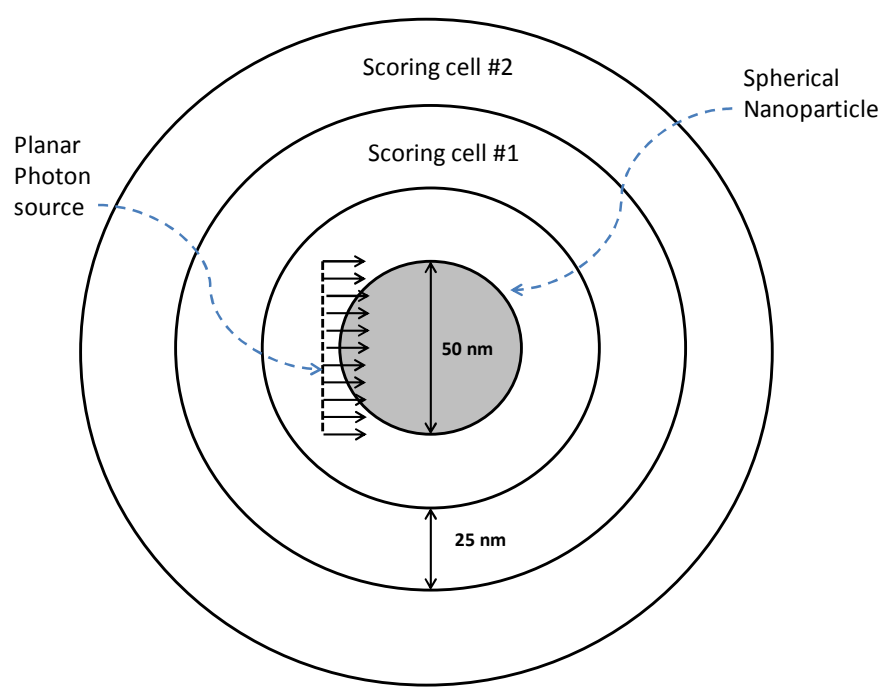

Figure 1. Schematic diagram of Monte Carlo simulated geometry 


\section{Results}

Figures 2-4 show the absorbed dose variation with distance from nanoparticle's surface with a step of $25 \mathrm{~nm}$ for five studied nanospheres and three photon energies. In Figure 2 absorbed dose from electrons with distance has been depicted for the photon energy of $30 \mathrm{keV}$. In Figure $\mathbf{2 A}$ as it can be seen the dose drops exponentially which shows itself as a straight line on a semi-log plot. It starts from around $10^{-3}$ at a distance of $37.5 \mathrm{~nm}$ and decreases to $10^{-9}-10^{-10}$ scale at 62.5 $\mathrm{nm}$. At the distance of $62.5 \mathrm{~nm}$, there is a breaking point for all nanoparticles. And, the absorbed dose is reduced at a slower pace with distance as it was shown in Figure 2B. In Figure 2B, the absorbed dose from 100 to $4000 \mathrm{~nm}$ has been shown. For example, the dose falls from $4.73 \times 10^{-9} \mathrm{~Gy}$ at $100 \mathrm{~nm}$ to $2.52 \times 10^{-12} \mathrm{~Gy}$ at $4000 \mathrm{~nm}$. Comparing the nanoparticles, iridium and gold deliver the higher doses and the same dosedistance pattern, while the bismuth is very close to them but it gets lower especially at long distances from nanoparticles surface. After them, hafnium and gadolinium nanoparticles deliver dose in descending order.

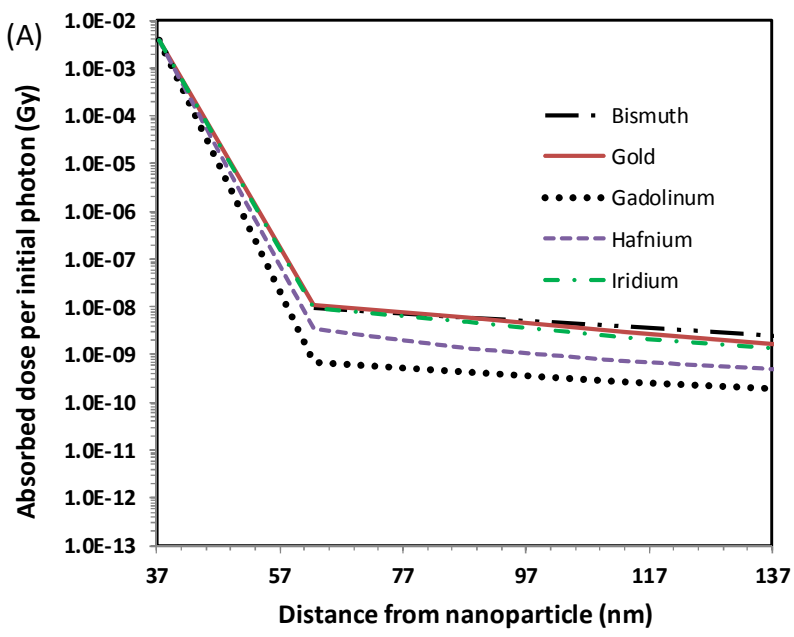

In Figure 3, the absorbed dose from electrons with distance has been depicted for the photon energy of $60 \mathrm{keV}$. In Figure 3A for the distance of 37.5 to $62.5 \mathrm{~nm}$ where a steep gradient of dose fall-off exists and like the $30 \mathrm{keV}$ case, the turning point for dose reduction locates at $62.5 \mathrm{~nm}$. The highest dose is seen for gadolinium and then bismuth nanoparticles. And the lowest dose was found for hafnium nanospheres. In Figure 3B the dose was depicted for 100 to $4000 \mathrm{~nm}$ and the curves follow a different pattern after $2000 \mathrm{~nm}$ compared to $30 \mathrm{keV}$. As can be seen that the bismuth and gadolinium, have comparable dose deposition properties till 2000nm. But after $2000 \mathrm{~nm}$ it reduces sharply and reaches hafnium at $2500 \mathrm{~nm}$.

In Figure 4, the calculated absorbed doses from secondary electrons with distance have been depicted for the photon energy of $100 \mathrm{keV}$. As can be seen in Figure 4A in short distances, gold and iridium nanospheres have the highest values which are very close together in terms of dose deposition with distance. While the lowest dose deposition was found for gadolinium.

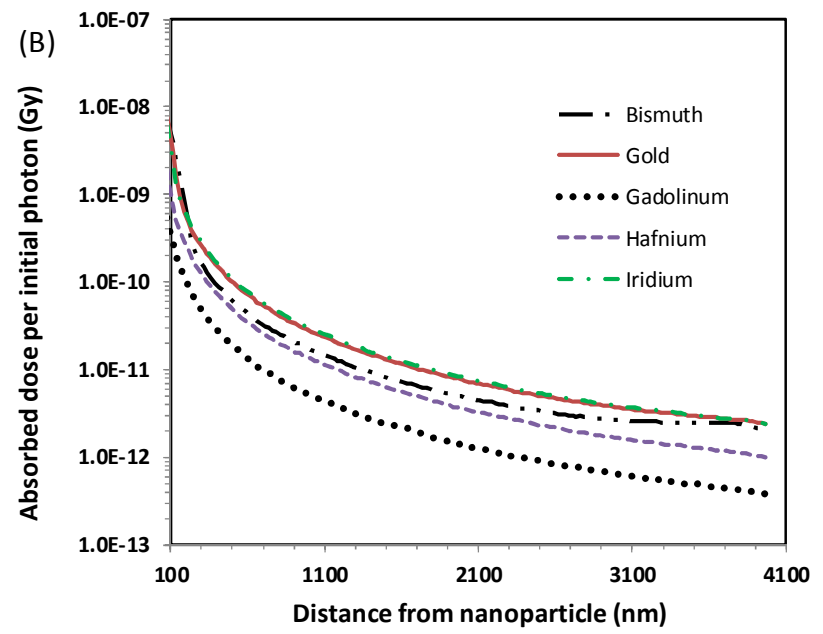

Figure 2. The calculated absorbed dose variation with distance from a single spherical nanoparticle with the diameter of $50 \mathrm{~nm}$ for impinging photons with energy of $30 \mathrm{keV}$. (A) At distances from 37.5 to $137.5 \mathrm{~nm}$ (B) at distances from 100 to $4000 \mathrm{~nm}$.
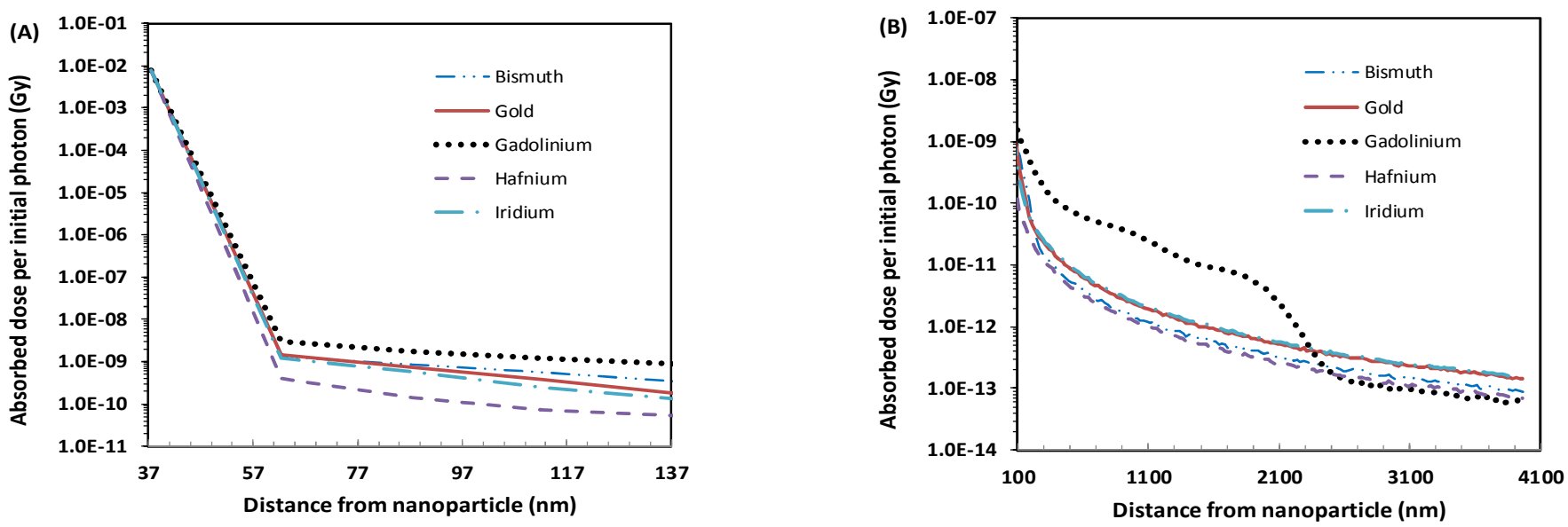

Figure 3. The calculated absorbed dose variation with distance from a single spherical nanoparticle with the diameter of $50 \mathrm{~nm}$ for impinging photons with energy of $60 \mathrm{keV}$. (A) at distances from 37.5 to $137.5 \mathrm{~nm}$ (B) at distances from $100 \mathrm{to} 4000 \mathrm{~nm}$. 

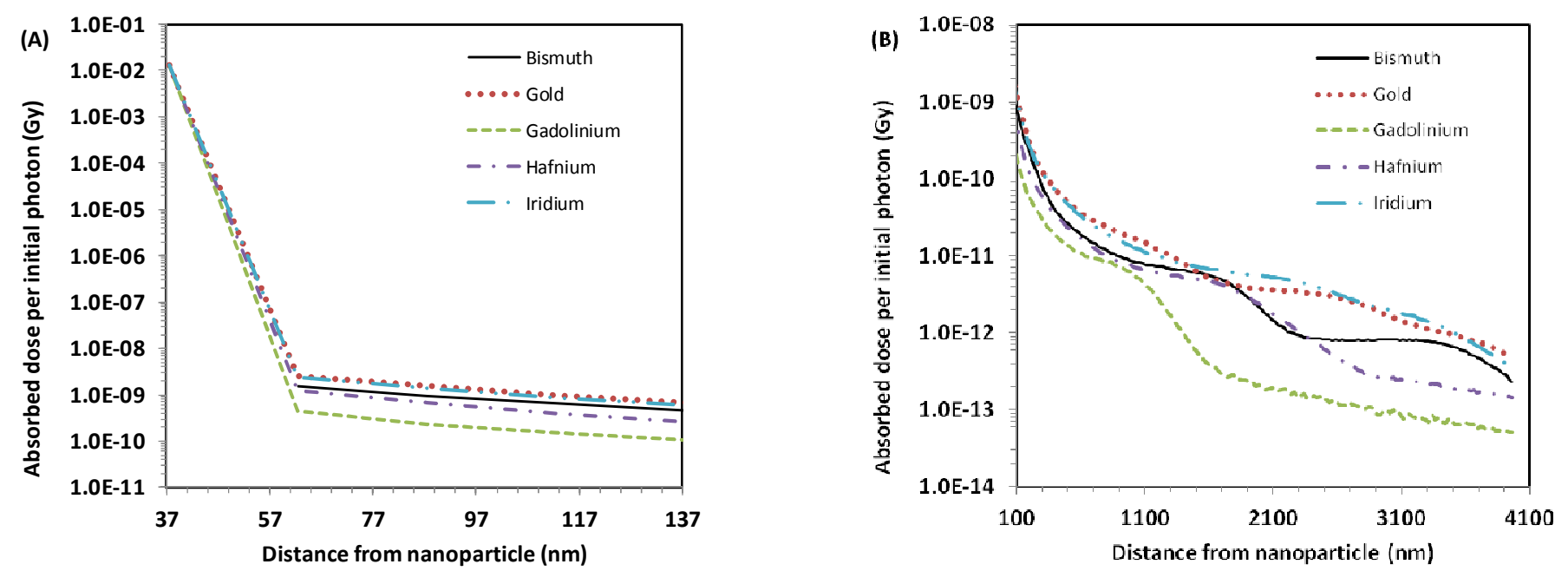

Figure 4. The calculated absorbed dose variation with distance from a single spherical nanoparticle with the diameter of $50 \mathrm{~nm}$ for impinging photons with energy of $100 \mathrm{keV}$. (A) at distances from 37.5 to $137.5 \mathrm{~nm}$ (B) at distances from 100 to $4000 \mathrm{~nm}$.

However, the same turning point of $62.5 \mathrm{~nm}$ was seen for this energy of photons like the previous energies. For long distances, gold and iridium keep their ranks compared to other nanospheres. But the dose deposition curves exhibits not a smooth and continuous reduction with distance and they fluctuate with distance for each nanoparticle.

Figure 5 illustrates the secondary electron flux per initial photon impinging on Bismuth, Gold, Gadolinium, Hafnium, and Iridium nanospheres. In Figure 5A where the results of 30 $\mathrm{keV}$ photons were shown, the electron flux for iridium and gold nanospheres was the highest among the studied atoms. While the electron flux for gadolinium showed the lowest value. For photon energy of $60 \mathrm{keV}$, the gadolinium had the highest secondary electron flux up to $1800 \mathrm{~nm}$. Then it went down rapidly and at the distance of $2200 \mathrm{~nm}$ has the lowest electron flux among the studied nanospheres. But for other nanospheres, the electron flux decreased continuously without sudden variations like gadolinium with distance. Up to 2000 $\mathrm{nm}$, hafnium and bismuth have the lowest secondary electron flux among studied nanoparticles.

Figures 6-8 show electron energy spectra for photon energies of 30, 60, $100 \mathrm{keV}$ respectively. In Figure 6, the secondary electron spectra were illustrated for $30 \mathrm{keV}$ photons. As can be seen there three groups of electrons in the energy spectra. The first group consists of the auger and multiply scattered electrons from $1 \mathrm{keV}$ to $12 \mathrm{keV}$, and the second and third groups are photoelectrons from $\mathrm{M}$ - and L- shells. In Figures 7 and 8, the energy spectra of secondary electrons have been depicted for 60 and $100 \mathrm{keV}$ photons. The location of low-energy auger electrons does not change considerably, but due to higher photon energy, the energy of photoelectrons is increased and other peaks such as auger electrons from $\mathrm{K}$ shell are seen in the spectra.
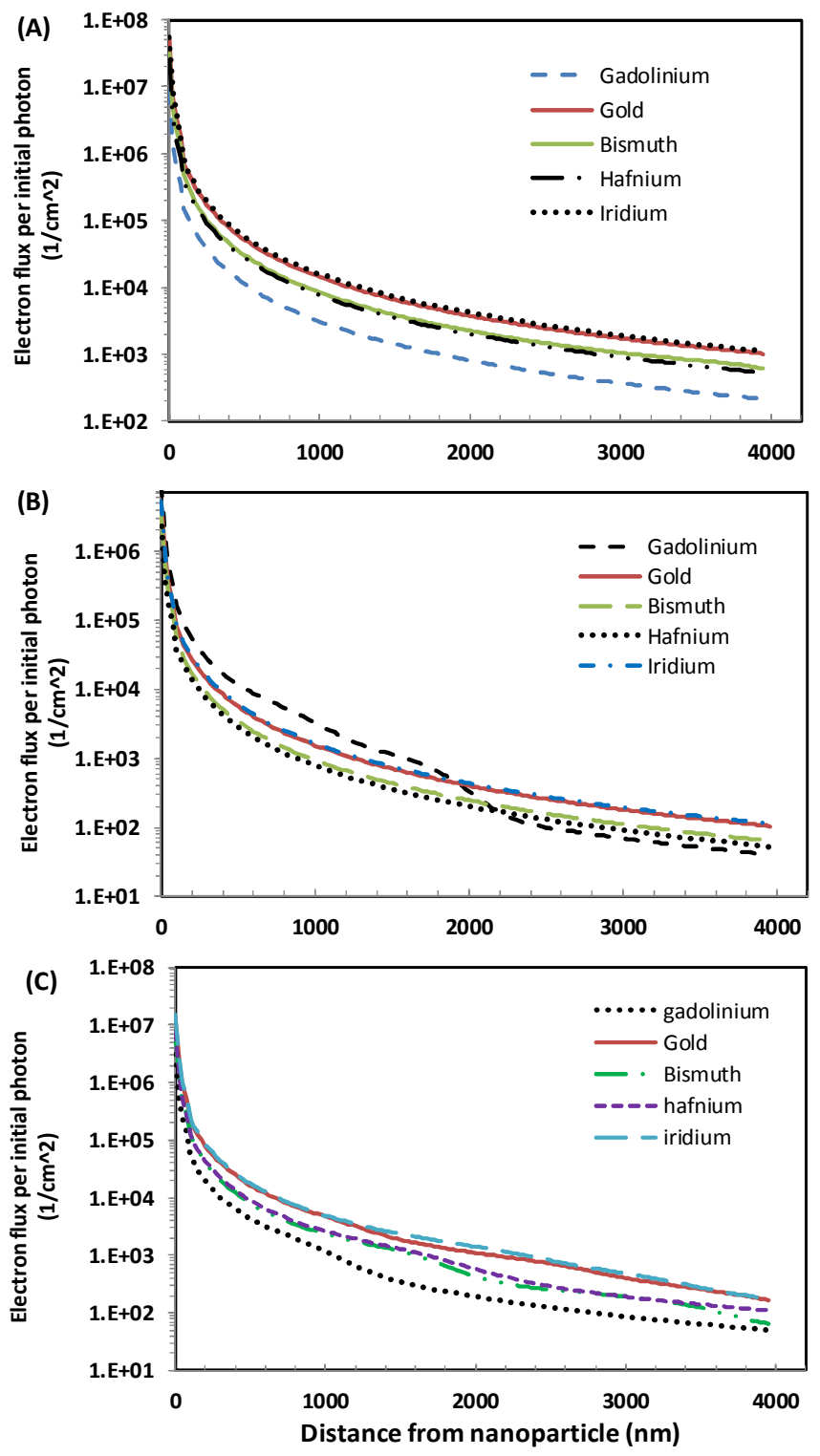

Figure 5. The calculated electron fluence with distance from the nanoparticle for three different energies of photons. (A) $30 \mathrm{keV}$ (B) $60 \mathrm{keV}$ (C) $100 \mathrm{keV}$ 

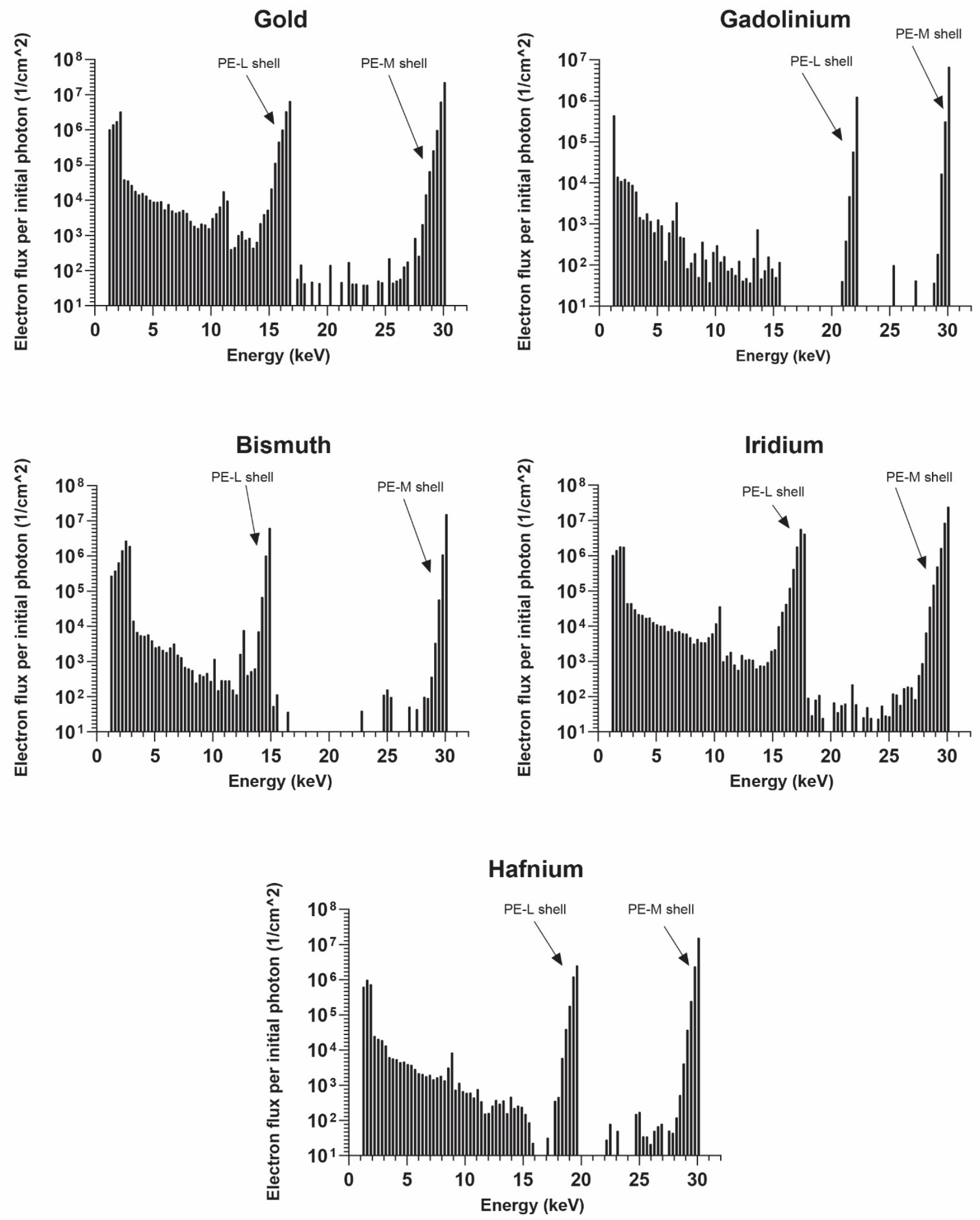

Figure 6. Energy spectra of secondary electrons exiting the nanoparticle surface for photon beam of $30 \mathrm{keV}$. 

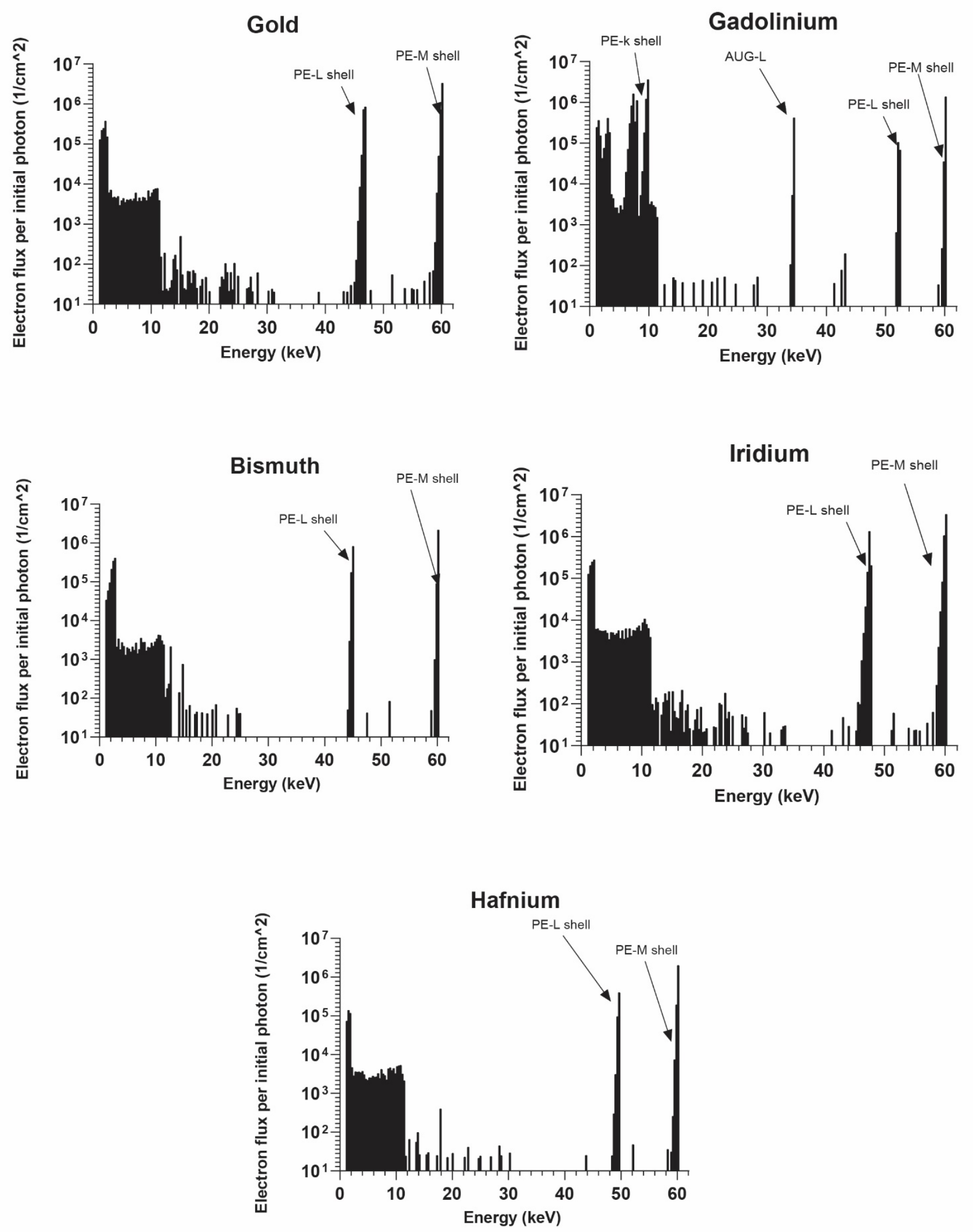

Figure 7. Energy spectra of secondary electrons exiting the nanoparticle surface for photon beam of $60 \mathrm{keV}$. 

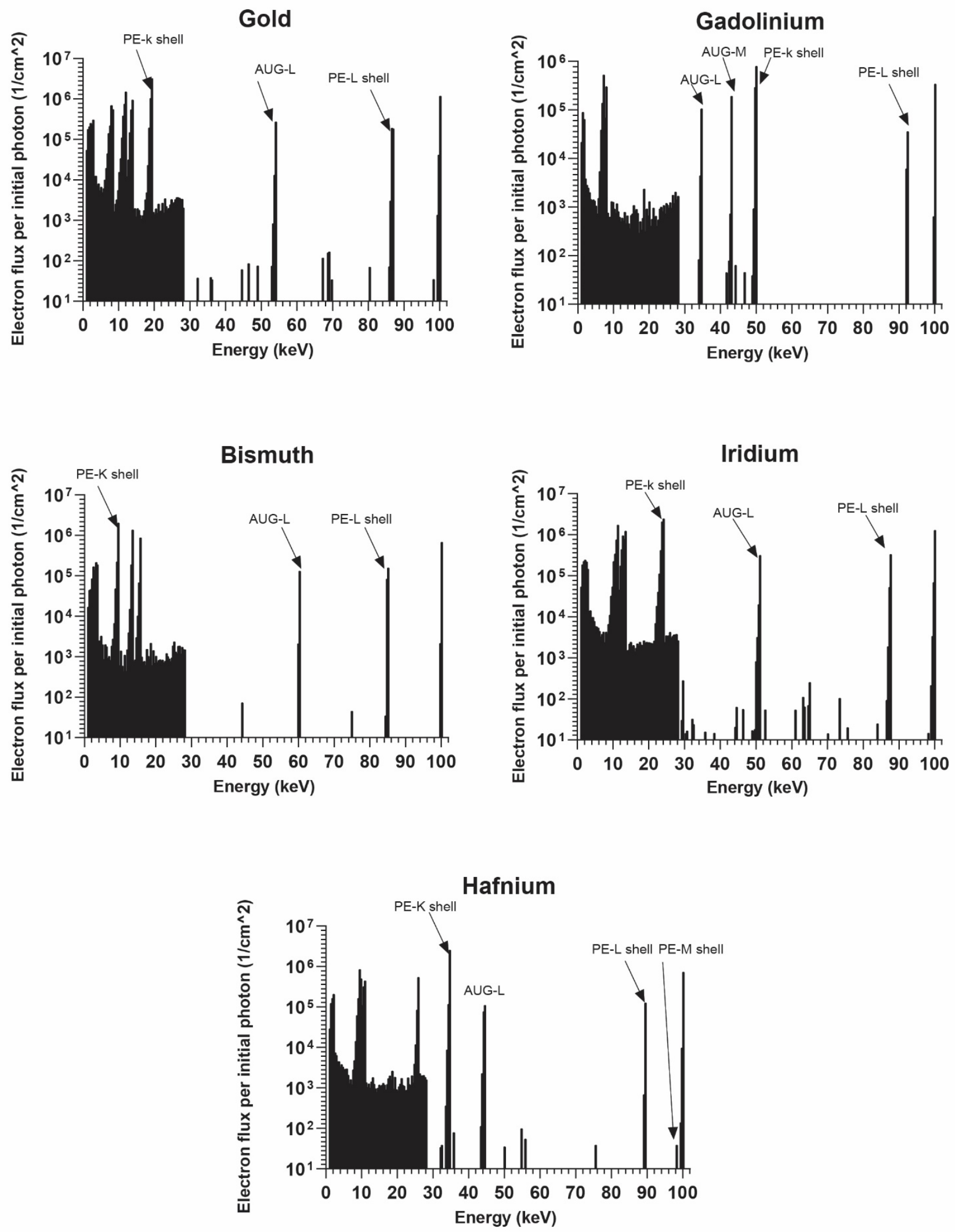

Figure 8. Energy spectra of secondary electrons exiting the nanoparticle surface for photon beam of $100 \mathrm{keV}$. 


\section{Discussion}

Our results were in line with the results of McMahon et al. ${ }^{33}$ as it was reported that secondary electrons were composed of photoelectrons and auger electrons with an energy around 10 $\mathrm{keV}$ with many less than $1 \mathrm{keV}$. However, due to the electron energy cutoffs of $1 \mathrm{keV}$ for the MCNPX code, we did not score auger less than $1 \mathrm{keV}$ in our simulations. On the other hand, their study indicated that compared to photoelectrons, auger electrons were more effective components in dose enhancement around nanoparticles up to $200 \mathrm{~nm}$ for gold nanoparticle with a diameter of $20 \mathrm{~nm}$ and photon energy of 40 $\mathrm{keV}$. Our finding was in accordance with their results. In

Figures 2-4, there was a higher dose deposition region up to 60 $\mathrm{nm}$, which can be attributed to low energy auger electrons, which diminished rapidly with distance, and then high energy photoelectrons are the prominent component of dose deposition around nanoparticles. And, according to McMahon et al only auger electrons of L-shell contribute to the dose deposition beyond $200 \mathrm{~nm}$ distance from nanoparticle surface. The observed differences in dose deposition around nanoparticles can be attributed to the electron energy cutoffs used in two studies and also differences in electron transport algorithms and libraries between MCNPX and GEANT codes.

The atomic number of an element impacts on K- and L-shell binding energy of its electrons and if the photon energy is slightly higher than electron binding energy, the probability of photoelectric interaction will be significantly high. However, photoelectric interaction with other shells such as $\mathrm{M}$ has a lower probability. Our results indicated that the deposition of energy around the NP is higher near its surface and within the distance of $100 \mathrm{~nm}$, it hastily drops by nearly an order of magnitude.

In Figures 2-4, it was shown the very strong dose gradients around nanoparticles up to $60 \mathrm{~nm}$ from nanoparticle surface, which was independent of photon energy and slightly varied with nanoparticle type. For gadolinium, it is in agreement with the finding of Delorme et al. ${ }^{11}$ which showed the same steep gradient for small nanoparticles as small as $1 \mathrm{~nm}$ and it was extended to less than $100 \mathrm{~nm}$ for nanoparticle with a diameter of $50 \mathrm{~nm}$.

The electron flux with distance curves indicated a close similarity between dose-distance and electron flux-distance for all energies and nanoparticles. Thus, the electron flux variation with distance had the same scenario as dose deposition concerning the nanoparticle type and photon energy.

We obtained the electron energy spectra for five studied nanoparticles, in which the energy spectrum for gadolinium was consistent with the results of published articles. ${ }^{11,12,34}$ The origin of peaks in all calculated electron energy spectra was identified using atomic relaxation tables and their binding energies. In an in silico and in vitro study by Delorme et al., the gadolinium nanoparticles were used for radio-sensitization in a range of photon energies from $30 \mathrm{keV}$ to $1.25 \mathrm{MeV}$. The simulations' results were not in agreement with the in vitro finding considering the photon energy effect. However, in silico results showed that maximum DEF found at $65 \mathrm{keV}$ and $58 \mathrm{keV}$ for nucleus and cytoplasm respectively. Besides, optimal X-ray energy above Gd K-edge was a key factor for increased dose around nanoparticle for photons in the $\mathrm{keV}$ range. Our finding was in complete agreement with their modeling results and gadolinium showed higher dose deposition for the photon energy of $60 \mathrm{keV}$.

In another study by Maggiorella et al. ${ }^{14}$ which was done on hafnium oxide nanoparticles and their application in radiation therapy, they used photons in $\mathrm{keV}$ and $\mathrm{MeV}$ ranges for both $\mathrm{MC}$, in vitro and in vivo studies. They did not provide any information regarding the nano-scaled dosimetric features of hafnium oxide nanoparticles, but they showed its considerable $\mathrm{DEF}$ for the $200 \mathrm{keV}$ photon beam. We were not able to compare our results with their findings.

As it was stated by other studies the Auger electrons are effective components in dose deposition around small nanoparticles. In the current study, for the nanoparticle with the diameter of $50 \mathrm{~nm}$, the effect of auger electrons in dose deposition was significant around nanoparticle which was in agreement with the results of McMahon et al. ${ }^{33}$, but in contrast with another study by Douglass et al. ${ }^{35}$ which showed that auger electron contribution in dose was insignificant. This discrepancy arises from the size of nanoparticle of $400 \mathrm{~nm}$ which was used in their study.

Comparing the nanoparticles showed promising potentials for iridium nanoparticles in enhancing the dose of low energy photons which was comparable to gold and bismuth in some photon energies. Bismuth and gold had a higher dose deposition for the energies of 30 and $100 \mathrm{keV}$ photon beams due to the proximity of photon energies to their L- and Kelectrons and the higher probability of photoelectric effect in these energies.

\section{Conclusion}

The application of high atomic number (Z) nanoparticles in radiation therapy has been proposed as a promising method for the treatment of solid cancers. Our finding showed a promising potential for iridium nanoparticles in dose enhancement of low energy photons which was comparable to gold and bismuth. Also, the gadolinium nanoparticle was very effective in dose enhancement for the photon energy of $60 \mathrm{keV}$. The dose deposition in the nano-scale was dependent on the atomic number of nanoparticle as well as photon energy. Thus, it can be concluded that for each photon energy, a suitable nanoparticle based on K-edge or L-edge binding energy of orbital electrons can augment the dose enhancement of nanoparticles irradiated by low energy photon beams used in brachytherapy. 


\section{Acknowledgments}

The project was conducted in the Molecular Medicine Research Center and Department of Medical Physics of Tabriz University of Medical Sciences.

\section{References}

1. Afkham Y, Mesbahi A, Alemi A, et al. Design and fabrication of a Nano-based neutron shield for fast neutrons from medical linear accelerators in radiation therapy. Radiat Oncol. 2020;15:105.

2. Ahmad R, Schettino G, Royle G, et al. Radiobiological Implications of Nanoparticles Following Radiation Treatment. Part Part Syst Charact. 2020;37(4):1900411.

3. Badrigilan S, Shaabani B, Aghaji NG, Mesbahi A. Graphene Quantum Dots-Coated Bismuth Nanoparticles for Improved CT Imaging and Photothermal Performance. Int J Nanosci. 2020;19(1):18500453.

4. Badrigilan S, Shaabani B, Gharehaghaji N, Mesbahi A. Iron oxide/bismuth oxide nanocomposites coated by graphene quantum dots: Three-in-one theranostic agents for simultaneous CT/MR imaging-guided in vitro photothermal therapy. Photodiagn Photodyn Ther. 2019;25:504-514

5. Ghasemi-Jangjoo A, Ghiasi H. Monte Carlo study on the gold and gadolinium nanoparticles radio-sensitizer effect in the prostate 125I seeds radiotherapy. Pol J Med Phys Eng. 2019;25(3):165-169.

6. Kuncic Z, Lacombe S. Nanoparticle radio-enhancement: Principles, progress and application to cancer treatment. Phys Med Biol. 2018;63:02TR01.

7. Mortezazadeh T, Gholibegloo E, Khoobi M, et al. In vitro and in-ávivo characteristics of doxorubicin-loaded cyclodextrine-based polyester modified gadolinium oxide nanoparticles: a versatile targeted theranostic system for tumour chemotherapy and molecular resonance imaging. J Drug Targeting. 2020;28(5):533-546.

8. Pirayesh Islamian J, Hatamian M, Aval NA, et al. Targeted superparamagnetic nanoparticles coated with 2-deoxy-D-gloucose and doxorubicin more sensitize breast cancer cells to ionizing radiation. Breast. 2017;33:97-103.

9. Sadeghian M, Akhlaghi P, Mesbahi A. Investigation of imaging properties of novel contrast agents based on gold, silver and bismuth nanoparticles in spectral computed tomography using Monte Carlo simulation. Pol J Med Phys Eng. 2020;26:21-29.

10. Yazdani P, Mansouri E, Eyvazi S, et al. Layered double hydroxide nanoparticles as an appealing nanoparticle in gene/plasmid and drug delivery system in C2C12 myoblast cells. Artif Cells Nanomed Biotechnol. 2019;47(1):436-442.

11. Delorme R, Taupin F, Flaender M, et al. Comparison of gadolinium nanoparticles and molecular contrast agents for radiation therapy-enhancement. Med.Phys. 2017;44(11):5949-5960.

12. Hwang C, Kim JM, Kim J. Influence of concentration, nanoparticle size, beam energy, and material on dose enhancement in radiation therapy. J Rad Res. 2017;58(4):405-411.

13. Jangjoo AG, Ghiasi H, Mesbahi A. A Monte Carlo study on the radio-sensitization effect of gold nanoparticles in brachytherapy of prostate by 103Pd seeds. Pol J Med Phys Eng. 2019;25(2):87-92.

14. Maggiorella L, Barouch G, Devaux C, et al. Nanoscale radiotherapy with hafnium oxide nanoparticles. Future Oncol. 2012;8:11671181.

15. Matsumoto K, Saitoh H, Doan TLH, et al. Destruction of tumor mass by gadolinium-loaded nanoparticles irradiated with monochromatic X-rays: Implications for the Auger therapy. Sci Rep. 2019;9:13275.

16. McMahon SJ, Mendenhall MH, Jain S, Currell F. Radiotherapy in the presence of contrast agents: A general figure of merit and its application to gold nanoparticles. Phys Med Biol .2008;53:5635-5651.

17. Mesbahi A. A review on gold nanoparticles radiosensitization effect in radiation therapy of cancer. Rep Pract Oncol Radiother. 2010;15(6):176-180.

18. McMahon SJ, Hyland WB, Muir MF,. Erratum: Biological consequences of nanoscale energy deposition near irradiated heavy atom nanoparticles. (Scientific Reports). Sci Rep. 2013;3:1725.

19. Verry C, Porcel E, Chargari C, et al. Use of nanoparticles as radiosensitizing agents in radiotherapy: State of play. Cancer Radiother. 2019;23(8):917-921.

20. Carter JD, Cheng NN, Qu Y, et al. Nanoscale energy deposition by X-ray absorbing nanostructures. J Phys Chem B. 2007;111(40):11622-11625

21. Casta R, Champeaux JP, Cafarelli P, et al. Model for electron emission of high-Z radio-sensitizing nanoparticle irradiated by X-rays. J Nanopart Res. 2014;16:2480.

22. Casta R, Champeaux JP, Sence M, et al. Comparison between gold nanoparticle and gold plane electron emissions: a way to identify secondary electron emission. Phys Med Biol. 2015;60(23):9095-9105 
23. Casta R, Champeaux KP, Sence M, et al. Gold nanoparticle electron and photon emissions after X-ray absorption. J Phys Conf Ser. 2015;635(10):102018.

24. Leung MKK, Chow JCL, Chithrani BD, et al. Irradiation of gold nanoparticles by x-rays: Monte Carlo simulation of dose enhancements and the spatial properties of the secondary electrons production. Med Phys. 2011;38:624-631

25. Liu Y, Zhang P, Li F, et al. Metal-based NanoEnhancers for future radiotherapy: Radiosensitizing and synergistic effects on tumor cells. Theranostics. 2018;81824-1849.

26. Sancey L, Lux F, Kotb S, et al. The use of theranostic gadolinium-based nanoprobes to improve radiotherapy efficacy. Br J Radiol. 2014;87:20140134.

27. Taupin F, Flaender M, Delorme R, et al. Gadolinium nanoparticles and contrast agent as radiation sensitizers. Phys Med Biol. 2015;60(11):4449-4464.

28. Sherck NJ, Won YY. Technical Note: A simulation study on the feasibility of radiotherapy dose enhancement with calcium tungstate and hafnium oxide nano- and microparticles. Med Phys. 2017;44:6583-6588.

29. Botchway SW, Coulter JA, Currell FJ. Imaging intracellular and systemic in vivo gold nanoparticles to enhance radiotherapy. Br J Radiol. 2015;881054):20150170.

30. Tsiamas P, Liu B, Cifter F, et al. Impact of beam quality on megavoltage radiotherapy treatment techniques utilizing gold nanoparticles for dose enhancement. Phys Med Biol. 2013;58(3):451-464.

31. Villagomez-Bernabe B, Currell FJ. Physical Radiation Enhancement Effects Around Clinically Relevant Clusters of Nanoagents in Biological Systems. Sci Rep. 2019;9:8156.

32. Zangeneh M, Nedaei HA, Mozdarani H, et al. Enhanced cytotoxic and genotoxic effects of gadolinium-doped ZnO nanoparticles on irradiated lung cancer cells at megavoltage radiation energies. Mater Sci Eng C. 2019;103:109739.

33. McMahon SJ, Hyland WB, Muir MF, et al. Biological consequences of nanoscale energy deposition near irradiated heavy atom nanoparticles. Sci Rep. 2011;1:18. doi:10.1038/srep00018.

34. Jamil MZAM, Mohamed F, Rosli NRAM, et al. Effect of gamma irradiation on magnetic gadolinium oxide nanoparticles coated with chitosan (GdNPs-Cs) as contrast agent in magnetic resonance imaging. Radiat Phys Chem. 2019;165:108407.

35. Douglass M, Bezak E, Penfold S. Monte Carlo investigation of the increased radiation deposition due to gold nanoparticles using kilovoltage and megavoltage photons in a 3D randomized cell model. Med.Phys. 2013;40(7):071710. 